\title{
Analysis of Cylindrical Waveguide Discontinuities Using Vectorial Eigenmodes and Perfectly Matched Layers
}

\author{
Peter Bienstman, Student Member, IEEE, Henk Derudder, Roel Baets, Senior Member, IEEE, \\ Frank Olyslager, Senior Member, IEEE, and Daniël De Zutter, Fellow, IEEE
}

\begin{abstract}
In this paper, we analyze the scattering at discontinuities in cylindrical waveguides, starting from a vectorial eigenmode expansion and by introducing perfectly matched layer (PML) boundary conditions. The structure under study is enclosed in a metal cylinder to discretize the radiative mode spectrum, while the coating of this cylinder with PML vastly reduces the influence of parasitic reflections at the metal. This allows for a model that is both faster and more accurate than previous models.
\end{abstract}

Index Terms-Absorbing boundary conditions, mode matching, perfectly matched layers, scattering.

\section{INTRODUCTION}

$\mathbf{T}$ HE analysis of discontinuities in cylindrical waveguides is of great practical importance, not only to investigate the reflections at the end facets of optical fibers, but also as a subproblem in the study of vertical-cavity surface-emitting lasers (VCSELs). These cylindrical resonators consist of a stack of epitaxially grown dielectric layers, forming a top and bottom distributed reflector around a light-emitting active layer (Fig. 1). The laser light exits the resonator perpendicular to the direction of the layers. These cavities can be considered as a sequence of cylindrical waveguides, each having a different thickness and cross section [1], [2].

The advantage of an eigenmode expansion [3], as compared to other methods based on spatial discretization [4]-[6], is that the field is represented by a sum of a relatively small number of eigenmodes, as compared to being specified at a large number of grid points on a discrete mesh. The eigenmode formulation, therefore, introduces a significantly smaller number of unknowns, leading to a greatly reduced computational effort.

In order to accurately model diffraction effects, it is necessary to include both guided and radiation modes in the eigenmode expansion. In open structures, however, the radiation modes form a continuum that is hard to model. Therefore, the structures under study are typically placed inside a metal volume that discretizes

Manuscript received October 4, 1999. This work was supported in part under the Belgian Project Inter University Attraction Pole IUAP IV-13. The work of P. Bienstman was supported by the Flemish National Fund for Scientific Research under a doctoral fellowship. The work of F. Olyslager was supported by the Flemish National Fund for Scientific Research under a post-doctoral fellowship.

The authors are with the Department of Information Technology, Interuniversity Microelectronics Centre, Ghent University, B-9000 Gent, Belgium (e-mail Peter.Bienstman@rug.ac.be).

Publisher Item Identifier S 0018-9480(01)01076-6.

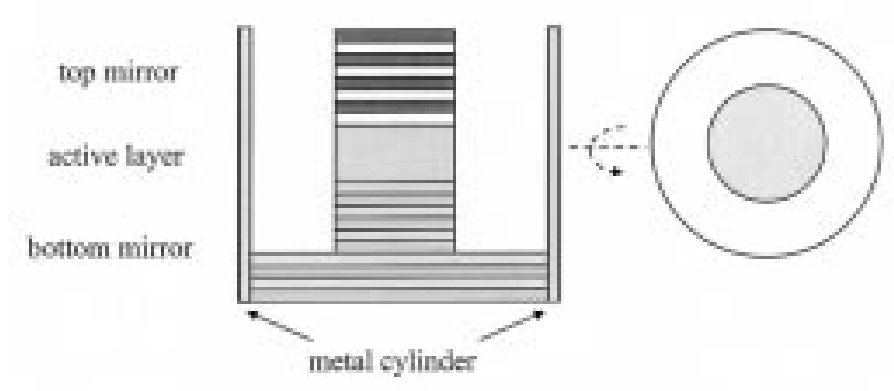

Fig. 1. Typical VCSEL structure, enclosed in a metal discretization cylinder.

the mode spectrum. However, this has the disadvantage of introducing parasitic reflections at the metal walls. Placing the metal walls sufficiently far from the structure of interest can minimize the effect of these reflections, but this, in turn, requires a larger number of modes for convergence.

Recently [7], [8], it was shown that coating the metal walls with a perfectly matched layer (PML) greatly reduces the influence of parasitic reflections in the case of planar slab waveguides. In this paper, this approach is extended to cylindrical geometries. It is shown that the use of a PML-coated discretization volume is able to accurately model open space, while at the same time requiring only a discrete set of modes.

This paper is organized as follows. Section II investigates the nature of the modes of an optical fiber-like structure enclosed in a metal cylinder coated with a PML. Section III computes the scattering at an interface between two different waveguides based on the well-known mode-matching technique. It is demonstrated that the use of a PML can greatly improve the accuracy and computation speed of the method. Section IV deals with the efficient localization of the propagation constants in the complex plane. Finally, in Section V, the tradeoffs are discussed in the choice of PML parameters. In the following, a $e^{j \omega t}$ time dependence is assumed.

\section{EIGENMODES OF A PML-COATED WAVEGUIDE}

We use the complex coordinate stretching formulation of PMLs [9], which states that an analytic continuation of Maxwell's equations onto complex coordinates provides for reflectionless absorption of incident waves, regardless of wavelength, incidence angle, or polarization. More specifically, 


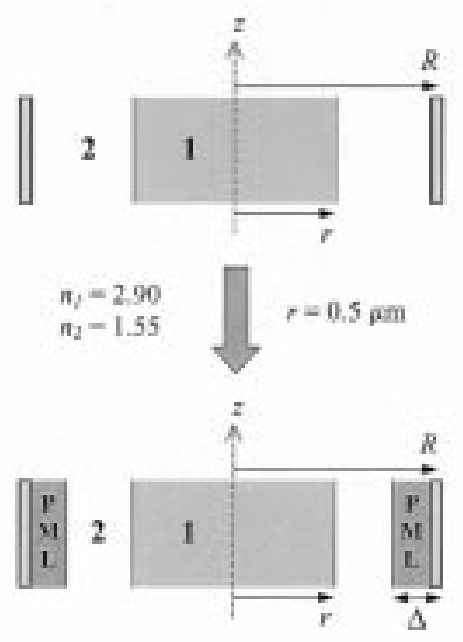

Fig. 2. Oxide aperture waveguide, enclosed in a metal cylinder (top) and a metal cylinder coated with a PML (bottom).

we introduce the following change of variables for the radial coordinate $\rho$ in cylindrical coordinate systems:

$$
\tilde{\rho}=\int_{0}^{\rho} s\left(\rho^{\prime}\right) d \rho^{\prime}
$$

with

$$
\left\{\begin{array}{l}
s(\rho)=1, \quad \text { for } \rho<R-\Delta \\
s(\rho)=1-j \alpha, \quad \text { for } R-\Delta<\rho<R .
\end{array}\right.
$$

Here, $R$ is the real radius of the metal cylinder and $\Delta$ is the real thickness of the PML. This change of variables recasts Maxwell's equation into ordinary-looking Maxwell's equations, but in a complex coordinate system. With the inclusion of the proper scaling factors for the fields inside the PML, this formulation is equivalent with the use of an active anisotropic medium as a perfectly absorbing boundary condition [10].

The advantage of the complex coordinate stretching is that existing closed-form solutions of Maxwell's equations can be easily transformed to include a PML, simply by allowing coordinates to assume complex values. For example, in the dispersion relation for a cylindrical waveguide enclosed in a metal cylinder, we simply have to add an imaginary part to the cylinder radius in order to include the presence of a PML that is coating this cylinder.

The dispersion relation for the waveguide in Fig. 2 (see, e.g., [11]) can be written as follows:

$$
\begin{aligned}
& {\left[r k_{\rho, 1} k_{\rho, 2}\right]^{2}\left[k_{\rho, 1} \mu_{r, 2} J_{n}\left(k_{\rho, 1} r\right) a_{\mathrm{TE}}-k_{\rho, 2} \mu_{r, 1} J_{n}^{\prime}\left(k_{\rho, 1} r\right) b_{\mathrm{TE}}\right]} \\
& \quad \cdot\left[k_{\rho, 1} \varepsilon_{r, 2} J_{n}\left(k_{\rho, 1} r\right) a_{\mathrm{TM}}-k_{\rho, 2} \varepsilon_{r, 1} J_{n}^{\prime}\left(k_{\rho, 1} r\right) b_{\mathrm{TM}}\right] \\
& \quad-\left[n \beta J_{n}\left(k_{\rho, 1} r\right) k_{0}\left(\varepsilon_{r, 1} \mu_{r, 1}-\varepsilon_{r, 2} \mu_{r, 2}\right)\right]^{2} b_{\mathrm{TE}} b_{\mathrm{TM}}=0
\end{aligned}
$$

with

$$
\left\{\begin{aligned}
a_{\mathrm{TE}}= & J_{n}^{\prime}\left(k_{\rho, 2} \tilde{R}\right) H_{n}^{(1)^{\prime}}\left(k_{\rho, 2} r\right) \\
& -H_{n}^{(1)^{\prime}}\left(k_{\rho, 2} \tilde{R}\right) J_{n}^{\prime}\left(k_{\rho, 2} r\right) \\
b_{\mathrm{TE}}= & J_{n}^{\prime}\left(k_{\rho, 2} \tilde{R}\right) H_{n}^{(1)}\left(k_{\left.\rho, 2^{r}\right)}\right. \\
& -H_{n}^{(1)^{\prime}}\left(k_{\rho, 2} \tilde{R}\right) J_{n}\left(k_{\rho, 2} r\right) \\
a_{\mathrm{TM}}= & J_{n}\left(k_{\rho, 2} \tilde{R}\right) H_{n}^{(1)^{\prime}}\left(k_{\rho, 2^{r}}\right) \\
& -H_{n}^{(1)}\left(k_{\rho, 2} \tilde{R}\right) J_{n}^{\prime}\left(k_{\rho, 2^{r}}\right) \\
b_{\mathrm{TM}}= & J_{n}\left(k_{\rho, 2} \tilde{R}\right) H_{n}^{(1)}\left(k_{\rho, 2} r\right) \\
& -H_{n}^{(1)}\left(k_{\rho, 2} \tilde{R}\right) J_{n}\left(k_{\rho, 2} r\right)
\end{aligned}\right.
$$

and

$$
\left\{\begin{array}{l}
k_{\rho, 1}=\sqrt{k_{0}^{2} \varepsilon_{r, 1} \mu_{r, 1}-\beta^{2}} \\
k_{\rho, 2}=\sqrt{k_{0}^{2} \varepsilon_{r, 2} \mu_{r, 2}-\beta^{2}}
\end{array} .\right.
$$

Here, $\beta$ is the propagation constant of the eigenmode in the $z$-direction and $k_{0}$ is the free-space wavenumber. The subscripts 1 and 2 refer, respectively, to the inner and outer regions, $k_{\rho, i}$ is the radial part of wavevector in medium $i$, and $\varepsilon_{r, i}$ and $\mu_{r, i}$ are the relative permittivity and permeability in medium $i$. A prime denotes derivation with respect to the argument, $H_{n}^{(1)}$ are Hankel functions of the first kind, and $n$ refers to the order of the Bessel functions. The real radius of the inner waveguide is $r$, and $\tilde{R}=R_{\text {re }}+j R_{\text {im }}$ is the complex radius of the metal cylinder. Note that the thickness $\Delta$ of the PML and the imaginary part $\alpha$ of the scaling factor $s_{\rho}$ do not enter into the equations separately, but only combined through $\tilde{R}=\int_{0}^{R} s\left(\rho^{\prime}\right) d \rho^{\prime}$.

Fig. 3 shows the distribution of the propagation constants of the waveguide shown at the bottom of Fig. 2, both in the complex $n_{\text {eff-plane }}\left(n_{\text {eff }}=\beta / k_{0}\right)$ and in the complex $k_{\rho, 2}$-plane. The parameters are chosen to correspond to the technologically important case of an aluminum-oxide (AlOx) aperture, where an isolating low-index AlOx ring inside a VCSEL resonator both acts as a focusing element and as a current confining structure [1]. The core has a radius $r=0.5 \mu \mathrm{m}$ and consists of AlAs with refractive index $n_{1}=2.9$. The cladding is AlOx $\left(n_{2}=1.55\right)$ and the metal cylinder has a radius $\tilde{R}=1.0-0.1 j$ $\mu \mathrm{m}$. The wavelength is $1 \mu \mathrm{m}$, and the modes shown have Bessel order $n=1$.

It can be seen from Fig. 3 that the influence of a PML on the guided modes is negligible in this case. They are still located on their original place on the imaginary $k_{\rho, 2}$-axis. The radiation modes, on the other hand, are no longer distributed along the real $k_{\rho, 2}$-axis, but are located on four branches in the complex plane. In analogy with the open case [12], we define the polarization according to the sign of the real part of $T$ given by

$$
\begin{aligned}
T=\frac{2}{k_{\rho, 1}} \varepsilon_{r, 1} \mu_{r, 1} & \frac{J_{n}^{\prime}\left(k_{\rho, 1} r\right)}{J_{n}\left(k_{\rho, 1} r\right)} \\
& -\frac{1}{k_{\rho, 2}}\left[\varepsilon_{r, 1} \mu_{r, 2} \frac{a_{\mathrm{TE}}}{b_{\mathrm{TE}}}-\varepsilon_{r, 2} \mu_{r, 1} \frac{a_{\mathrm{TM}}}{b_{\mathrm{TM}}}\right] .
\end{aligned}
$$

We designate modes with a positive real part of $T \mathrm{EH}$, and modes with a negative real part of HE. For high enough radial mode numbers (i.e., far away from $k_{\rho, 2}=0$ ), the branch closest 


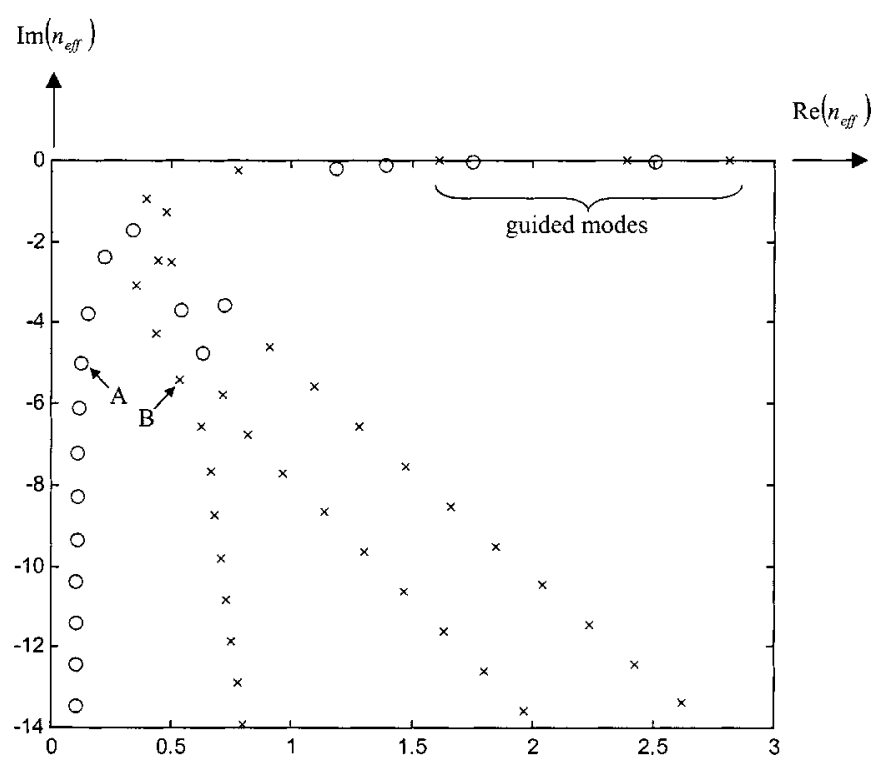

(a)

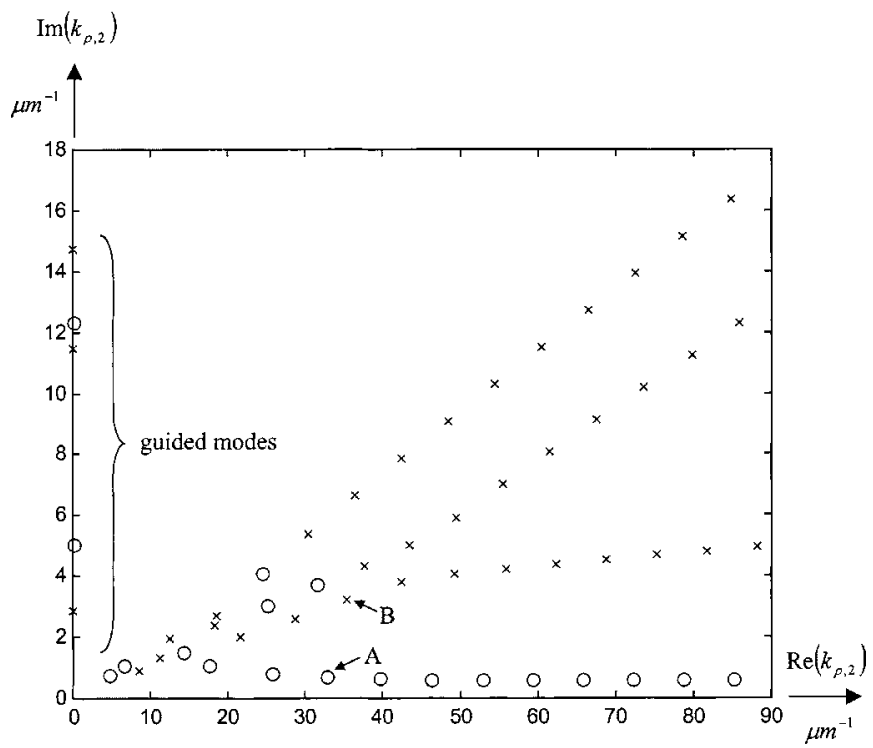

(b)

Fig. 3. (a) Distribution of propagation constants in the $n_{\text {cff }}$-plane (circles denote EH modes, crosses HE modes). (b) Distribution of radial propagation constants in the $k_{\rho, 2}$-plane (circles denote EH modes, crosses HE modes).

to the real $k_{\rho, 2}$-axis represents $\mathrm{EH}$ modes, while the other three branches consist of HE modes.

Fig. 4 shows the total electric field of the modes marked $A$ and $B$ on Fig. 3. The thickness $\Delta$ of the PML is chosen to be $0.25 \mu \mathrm{m}$, still with $\tilde{R}=1.0-0.1 j \mu \mathrm{m}$. Note that the field is significantly damped inside the PML. Therefore, by the time it reaches the metal wall, its amplitude has considerably decreased so that reflections will be much less pronounced. Also note that modes on the HE branches behave like surface waves, whose electric field is concentrated at the interface between the oxide and the PML.

For other Bessel orders $n$ the propagation factors still approximately lie along the same four branches: a single EH branch and three HE branches representing the PML surface modes. For $n=0$, the modes are, of course, no longer EH and HE

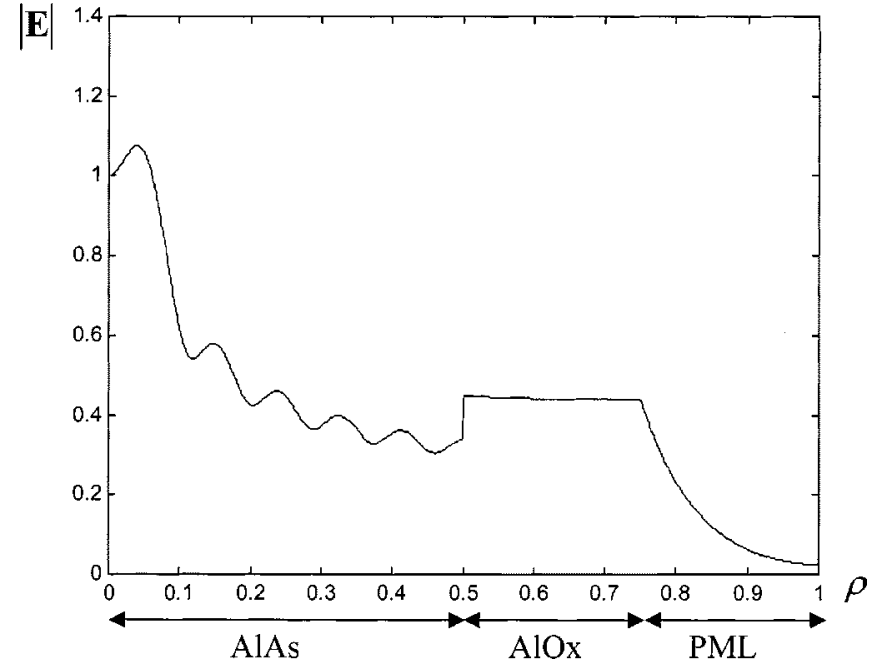

(a)

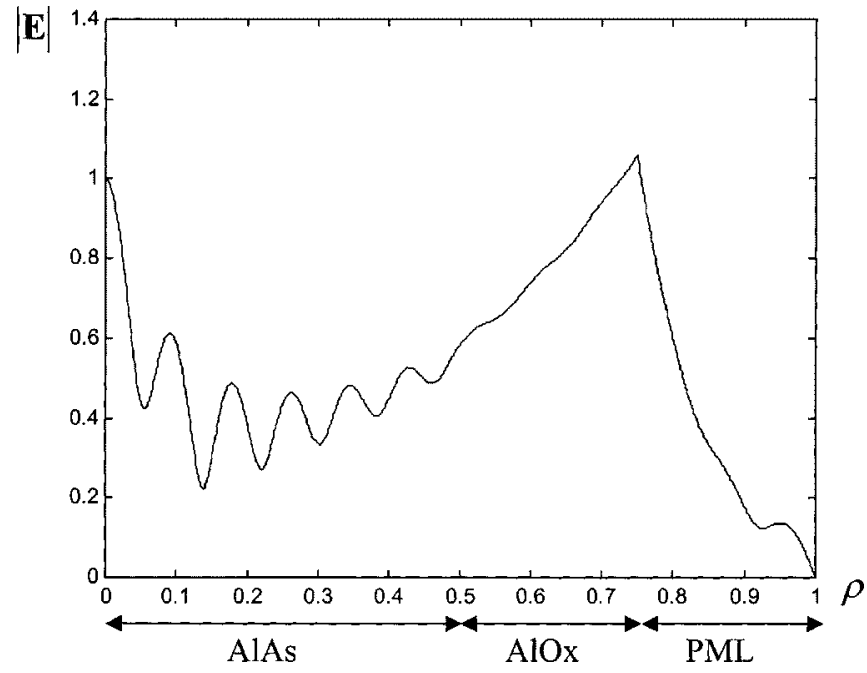

(b)

Fig. 4. (a) Total electric field of EH mode $A\left(n_{\text {cff }}=0.1208-4.9517 j\right)$. (b) Total electric field of HE mode $B\left(n_{\text {cff }}=0.5322-5.4249 j\right)$.

polarized, but the TM modes lie on the $\mathrm{EH}$ and the outer $\mathrm{HE}$ branch, while the TE modes are located on the two HE branches closest to the real $k_{\rho, 2}$-axis.

\section{SCATTERING AT AN ABRUPT INTERFACE}

To calculate the scattering at an abrupt interface, we use the well-known mode-matching technique [13]. This method starts out by imposing the continuity of the tangential-field components at the interface. Applying the orthogonality relations transforms the expressions into a set of linear equations, from which the scattering matrices can be calculated. Prominent in these expressions are overlap integrals over the cross section between the field profiles of mode $i$ from one waveguide and mode $j$ from the other waveguide [14]

$$
\int \mathbf{E}_{i} \times \mathbf{H}_{j} \cdot d \mathbf{S}
$$

These integrals can be calculated analytically, again by taking the same expressions valid in the case without PML and by al- 


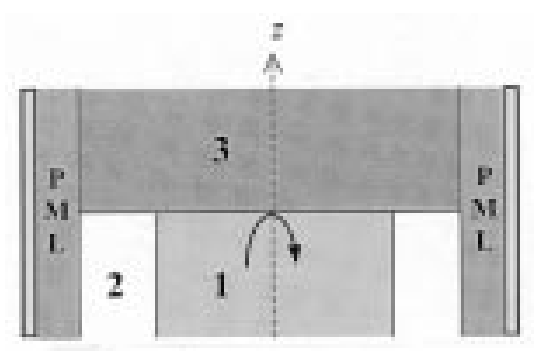

Fig. 5. Scattering of the fundamental mode from Fig. 2 at an interface with a uniform GaAs layer $(n=3.5)$.

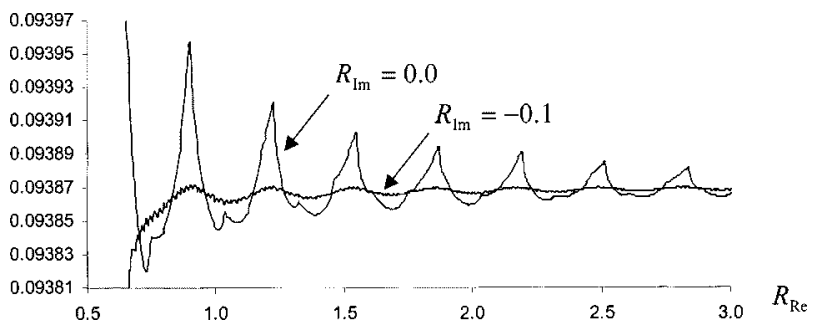

Fig. 6. Reflectivity of the fundamental mode of the incidence waveguide for the structure from Fig. 5.

lowing the radius of the metal cylinder to assume a complex value.

In order to model open space with this method, we have to pay attention to the reflections that occur at the metal cylinder. To illustrate the effectiveness of PML in reducing these parasitic reflections, we calculate the reflectivity onto itself of the fundamental mode of the waveguide from Fig. 2, at an interface with a uniform GaAs medium with refractive index 3.5 (Fig. 5). Fig. 6 shows the evolution of this reflectivity as a function of the real part of radius of the metal cylinder, both without a PML $\left(R_{\mathrm{im}}=0\right)$ and with a PML $\left(R_{\mathrm{im}}=-0.1\right)$.

Both curves converge to the same value, which is the reflectivity of the open problem, i.e., without the metal wall. In the presence of a PML, however, the reflection oscillations are significantly damped. This means that in order to achieve a given accuracy, we can use a significantly smaller metal cylinder in the PML case. This reduced computational volume requires fewer modes in order to achieve convergence. This can yield a large speedup in computation time, which scales roughly with the third power of the number of modes. The number of modes used in Fig. 6 is 115 times $R_{\text {re }} / \lambda$.

\section{EFFICIENT LOCATION OF COMPLEX ROOTS}

An important implementation question is how to efficiently locate the propagation constants in the complex plane. Should this step prove too time consuming, it could eliminate the speedup due to the smaller number of modes required.

A general technique for root finding in the complex plane is the so-called argument principal method (APM) [15]. This method uses contour integration to locate the zeros of an analytic function. Although this method works, it can be time consuming if a large number zeros is to be found.

Therefore, we opt for a different root-finding strategy. We start out from the lossless structure (i.e., without a PML) and calculate its guided and discretized radiation modes. These lie

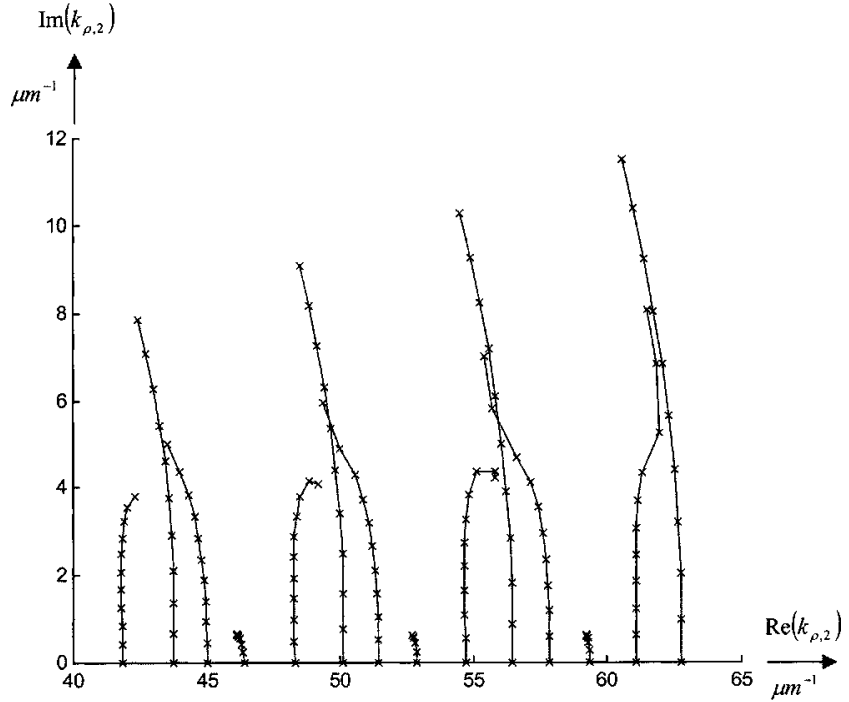

Fig. 7. Path traced out by the propagation constants of the radiation modes from Fig. 2, as $R_{\text {im }}$ changes from 0.0 to -0.1 . Every cross corresponds to a change in $R_{\text {im }}$ of 0.001 .

on the coordinate axes and can be calculated with relatively little numerical effort. In rare cases, a lossless structure can also support complex modes, which are not located on the coordinate axes [13]. These can be located by the APM, and since the number of complex modes is much smaller than the number of guided and radiation modes, this application of the APM has little numerical penalty.

Starting from this set of guided, radiation, and complex modes, we now gradually increase the losses in the structure (e.g., in $R_{\mathrm{im}}$ ) and track the modes as they move from the coordinate axes into the complex plane. This technique poses no numerical problems since two modes rarely become degenerate during the tracking process. The path traced out by some of these radiation modes is shown in Fig. 7, again for the waveguide structure from Fig. 2 with $R_{\mathrm{im}}=-0.1$.

This approach is significantly faster than the application of the APM to directly find all the modes in the lossy case.

The entire calculation of the scattering matrices, including root finding, takes under $3 \mathrm{~s}$ for 50 modes using a $250-\mathrm{MHz}$ Ultrasparc processor.

\section{CHOICE OF PML PARAMETERS}

When modeling open space by using a metal cylinder coated with a PML, one has two degrees of freedom: the real and imaginary parts of the cylinder radius.

Increasing the (absolute value of the) imaginary part $R_{\mathrm{im}}$ decreases the amplitude of the oscillations due to parasitic reflections. It also entails a small increase in computation time to locate these modes because they trace a longer path in the complex plane. For the simulations on the structure from Fig. $6,-0.1$ was found to be a good compromise.

Increasing the real part $R_{\text {re }}$ also increases the accuracy, but to a much smaller extent as compared to changing $R_{\mathrm{im}}$. Moreover, the numerical effort associated with increasing $R_{\text {re }}$ is much larger because of the larger computational volume and the higher number of modes required. On the other hand, 


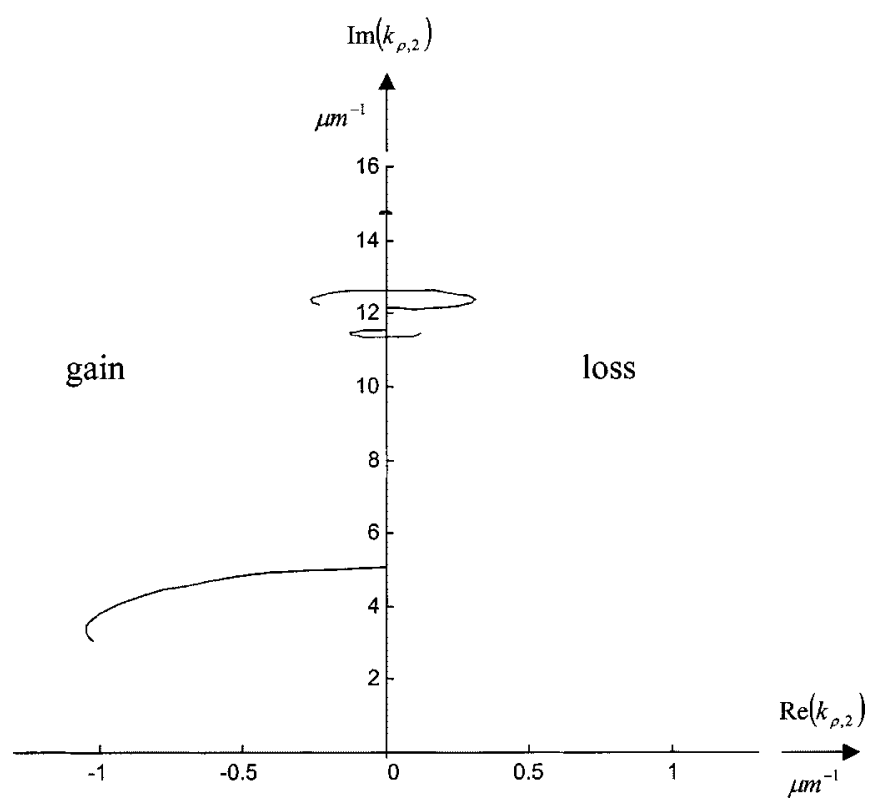

Fig. 8. Path traced out by the propagation constants of the guided modes from Fig. 2, as $R_{\text {im }}$ changes from 0.0 to -0.2 , but now with $R_{\mathrm{rc}}=0.55 \mu \mathrm{m}$.

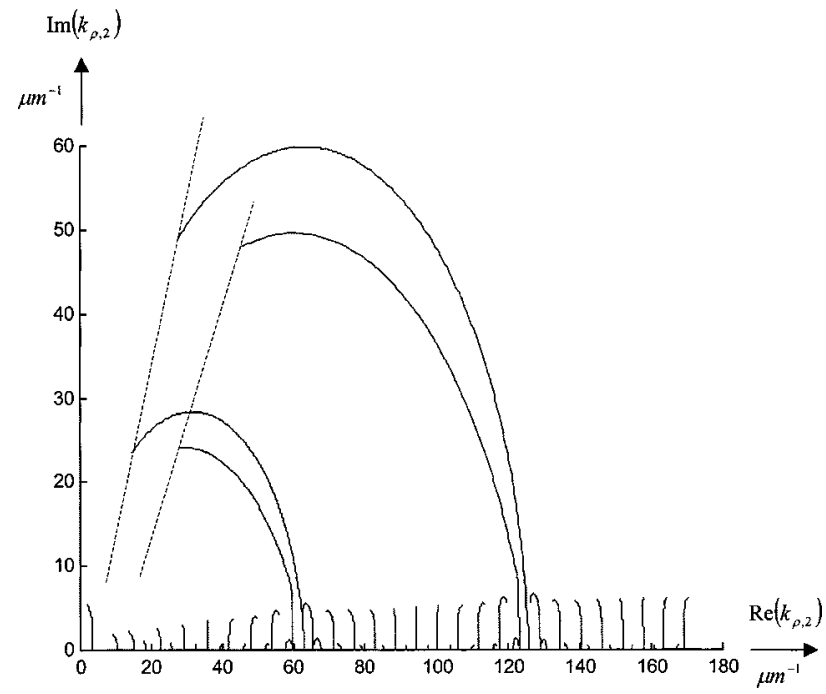

Fig. 9. Path traced out by the propagation constants of the radiation modes from Fig. 2, as $R_{\text {im }}$ changes from 0.0 to -0.1 , but now with $R_{\mathrm{re}}=0.55 \mu \mathrm{m}$. (dashed lines indicate outer HE branches).

$R_{\text {re }}$ should not be chosen too small either since the metal will then interact with the evanescent tails of the guided modes and strongly disturb the results. This is true even in the presence of a PML because the imaginary part of the radius is only effective in damping waves that are propagative in the direction normal to the PML, as is the case for radiation modes of the structure.

Fig. 8 illustrates the effect of a PML on the guided modes of the structure from Fig. 2, but now with $R_{\mathrm{re}}=0.55 \mu \mathrm{m}$. The path traced by a guided mode when changing $R_{\mathrm{im}}$ from 0.0 to -0.2 can take it through the lossy and/or gain quadrant. At first sight, it may seem surprising that some modes will experience gain in the presence of a PML, but this can be understood by looking at the anisotropic formulation of a PML [10]: the material tensor is that of a material with gain in some directions and loss in others.
This particular balance between loss and gain is precisely what achieves reflectionless absorption for propagative waves. For waves that are evanescent normal to the PML, this balance can either yield lossy or amplified modes. Also note that the effect is more pronounced for higher order guided modes, which are not so well confined to the core and are, therefore, more influenced by the PML.

A second reason why $R_{\text {re }}$ should not be chosen too small is illustrated in Fig. 9, which shows the path trace of the radiation modes for the structure of Fig. 2, with $R_{\text {re }}=0.55 \mu \mathrm{m}$ and $R_{\mathrm{im}}=-0.1$. The two outer HE branches (indicated by the dashed lines) are now only sparsely populated. This has implications when considering the convergence of the results as a function of the number of modes retained in the expansion. Since the number of modes on each branch should be taken sufficiently high, a large total number of modes is required in order to arrive at a large enough number of modes on the outer branches.

To summarize, $R_{\text {re }}$ should be chosen large enough so that the evanescent tails of the guided modes are sufficiently damped before reaching the metal. This also implies that the method is less practical for modes very near to cutoff.

\section{CONCLUSION}

In this paper, we have demonstrated an eigenmode expansion technique in cylindrical structures, where we used a PML to clad the discretization volume. The reflectionless absorption provided by the PML allows an efficient simulation of open space, while still maintaining the advantage of a discrete set of modes.

\section{REFERENCES}

[1] B. Demeulenaere, P. Bienstman, B. Dhoedt, and R. Baets, "Detailed study of AlAs-oxidized apertures in VCSEL cavities for optimized modal performance," IEEE J. Quantum Electron., vol. 35, pp. 358-367, Mar. 1999.

[2] P. Bienstman, B. Demeulenaere, B. Dhoedt, and R. Baets, "Simulation results of transverse-optical confinement in airpost, regrown, and oxidized vertical-cavity surface-emitting laser structures," J. Opt. Soc. Amer. B, Opt. Phys., vol. 16, no. 11, pp. 2055-2059, 1999.

[3] J. Willems, J. Haes, and R. Baets, "The bidirectional mode expansion method for two dimensional waveguides: The TM-case," Opt. Quantum Electron., vol. 27, no. 10, pp. 995-1007, 1995.

[4] G. R. Hadley, "Low-truncation-error finite difference equations for photonics simulation II: Vertical-cavity surface-emitting lasers," J. Lightwave Technol., vol. 16, pp. 142-151, Jan. 1998.

[5] M. J. Noble, J. P. Loehr, and J. A. Lott, "Quasi-exact optical analysis of oxide apertured microcavity VCSEL's using vector finite elements," IEEE J. Quantum Electron., vol. 34, pp. 2327-2339, Dec. 1998.

[6] B. Klein, L. F. Register, K. Hess, D. G. Deppe, and Q. Deng, "Self-consistent Green's function approach to the analysis of dielectrically apertured vertical-cavity surface-emitting lasers," Appl. Phys. Lett., vol. 73, no. 23, pp. 3324-3326, 1998.

[7] H. Derudder, F. Olyslager, and D. De Zutter, "Analysis of waveguide discontinuities using perfectly matched layers," Electron. Lett., vol. 34, no. 22, pp. 2138-2140, 1998.

[8] H. Derudder, F. Olyslager, D. De Zutter, and S. Van den Berghe, "Efficient mode-matching analysis of discontinuities in finite planar structures using perfectly matched layers," IEEE Trans. Antennas Propagat, submitted for publication.

[9] W. C. Chew, J. M. Jin, and E. Michielssen, "Complex coordinate stretching as a generalized absorbing boundary condition," Microwave Opt. Technol. Lett., vol. 15, no. 6, pp. 363-369, 1997.

[10] F. L. Teixeira and W. C. Chew, "Systematic derivation of anisotropic PML absorbing media in cylindrical and spherical coordinates," IEEE Microwave Guided Wave Lett., vol. 7, pp. 371-373, Nov. 1997. 
[11] K. A. Zaki and A. E. Atia, "Modes in dielectric-loaded waveguides and resonators," IEEE Trans. Microwave Theory Tech., vol. MTT-31, pp. 1039-1044, Dec. 1983.

[12] M. J. Lahart, "Analysis of a cylindrical dielectric waveguide with three regions by use of an invariant mode-definition parameter," J. Opt. Soc. Amer. A, Opt. Image Sci., vol. 15, no. 3, pp. 727-735, 1998.

[13] K. A. Zaki, S. W. Chen, and C. Chen, "Modeling discontinuities in dielectric-loaded waveguides," IEEE Trans. Microwave Theory Tech., vol. 36, pp. 1804-1810, Dec. 1988.

[14] F. Olyslager, Electromagnetic Waveguides and Transmission Lines. Oxford, U.K.: Oxford Univ. Press, 1999.

[15] L. M. Delves and J. N. Lynnes, "A numerical method for locating the zeros of an analytic function," Math. Comput., vol. 21, pp. 543-560, 1967.

Peter Bienstman (S'96) was born in Ghent, Belgium, in 1974. He received the electrical engineering degree from Ghent University, Gent, Belgium, in 1997, and is currently working toward the Ph.D. degree in electrical engineering at the Ghent University.

He is currently with the Department of Information Technology (INTEC), University of Gent. He has authored or co-authored several papers and has one patent pending. His research interests include the modeling of optical structures, notably resonant-cavity light-emitting diodes, VCSELs, and photonic-bandgap structures.

Mr. Bienstman is a member of the IEEE Lasers and Electro-Optics Society (IEEE LEOS) and the Flemish Engineers Association.

Henk Derudder was born in Kortrijk, Belgium, on January 8, 1973. He received the physical engineering degree from Ghent University, Gent, Belgium, in 1996, and is currently working toward the Ph.D. degree in information technology at Ghent University.

He is currently with the Department of Information Technology, University of Gent. His current research interest is the analysis of planar stratified waveguides in the presence of PMLs.

Roel Baets (M'88-SM'96) received the electrical engineering degree from Ghent University, Gent, Belgium, in 1980, the M.Sc. degree in electrical engineering from Stanford University, Stanford, CA, in 1981, and the Ph.D. degree from Ghent University, in 1984.

Since 1981, he has been with the Department of Information Technology (INTEL), University of Gent, where, since 1989, he has been a Professor. From 1990 to 1994, he was a Part-Time Professor at the Technical University of Delft, Delft, The Netherlands. He leads the Optoelectronic Components and Systems Group, University of Gent, Interuniversity Microelectronics Centre (IMEC), where he is involved with photonic devices for optical communication and optical interconnect. He has been involved in the field of III-V devices for optoelectronic systems. He has authored or co-authored approximately 200 publications and conference papers, and has made contributions to the design and fabrication of semiconductor laser diodes, passive guided wave devices, photonic integrated circuits (PICs), and microoptic components.

Dr. Baets is a member of the Optical Society of America, the IEEE Lasers and Electro-Optics Society (IEEE LEOS), the International Society for Optical Engineers (SPIE), and the Flemish Engineers Association. He is currently chairman of the IEEE Lasers and Electro-Optics (LEOS) Benelux chapter. He was a Program Committee member for the Optical Fibre Conference (OFC), European Conference on Optical Communications (ECOC), IEEE Semiconductor Laser Conference, ESSDERC, Conference on Lasers and Electro-Optics (CLEO) Europe, and the European Conference on Integrated Optics.
Frank Olyslager (S'90-M'93-SM'99) was born in 1966. He received the electrical engineering and Ph.D. degrees from Ghent University, Gent, Belgium, in 1989 and 1993, respectively.

He is currently a Senior Research Associate of the Fund for Scientific Research Flanders, Belgium, and a Professor at Ghent University. His research concerns different aspects of theoretical and numerical electromagnetics. $\mathrm{He}$ has authored or co-authored about 120 international journals and conference proceedings papers. He also authored Electromagnetic and Circuit Modeling of Multiconductor Transmission Lines (Oxford, U.K.: Oxford Univ. Press, 1993) and Electromagnetic Waveguides and Transmission Lines (Oxford, U.K.: Oxford Univ. Press, 1999). He is currently Associate Editor of Radio Science.

Dr. Olyslager was the recipient of the 1995 IEEE Microwave Prize.

Daniël De Zutter (M'92-SM'96-F'01) was born in 1953. He received the M.Sc. degree in electrotechnical engineering, the Ph.D. degree, and a degree equivalent to the French Aggregation or the German Habilitation from Ghent University, Gent, Belgium, in 1976, 1981, and 1984, respectively.

From 1976 to 1984 he was a Research and Teaching Assistant at Ghent University. From 1984 to 1996, he was with the National Fund for Scientific Research of Belgium. He is currently a Full Professor of electromagnetics at Ghent University. Most of his earlier scientific work dealt with the electrodynamics of moving media. His research now focuses on all aspects of circuit and electromagnetic modeling of high-speed and high-frequency interconnections, electromagnetic compatibility (EMC) and electromagnetic interference (EMI) topics, and indoor propagation. He has authored or co-authored approximately 80 international journal papers and 95 papers in conference proceedings. He co-authored Electromagnetic and Circuit Modeling of Multiconductor Transmission Lines (Oxford, U.K.: Oxford Univ. Press, 1993).

Dr. De Zutter is a member of the Electromagnetics Society. He was the recipient of the 1990 Montefiore Prize of the University of Liege and corecipient of the 1995 IEEE Microwave Prize Award presented by the IEEE Microwave Theory and Techniques Society (IEEE MTT-S) for the best publication in the field of microwaves. 